\title{
The Central Analytical Facility at the University of Alabama
}

\author{
R. L. Martens ${ }^{1}$, J. D. Goodwin ${ }^{1}$, R. A. Holler ${ }^{1}$, D.E. Nilkes ${ }^{2}$, and G.B. Thompson ${ }^{3}$ \\ ${ }^{1 .}$ Central Analytical Facility, University of Alabama, Tuscaloosa, AL 35487 \\ 2. Dept of Chemistry, University of Alabama, Tuscaloosa, AL 35487 \\ 3. Dept of Metallurgical \& Materials Engineering, University of Alabama, Tuscaloosa, AL 35487
}

The Central Analytical Facility (CAF) has a mission of service in support of the teaching, research and service for the University of Alabama (UA) [1]. Located in Tuscaloosa, Alabama, UA is the Capstone institution for the University of Alabama System [2]. The CAF has emerged as a première microanalytical and microstructural characterization facility within the Southeast region. Its primary focus is to assist on and off campus academic research institutions with their characterization needs. The CAF staff provides training and operational assistance in using the suite of characterization tools. Depending on need and frequency of visits, the staff can provide hands-on training to users for any and all equipment. The staff can also assist in addressing related questions on the capability of the instrumentation for your research needs. Instrument reservations and instrument billing rates, which help off-site service contract costs to ensure all tools are operational with minimal downtime, is handled through our Alabama Research Management System (ARMS). The following is a listing of the CAF instruments:

- Cameca's Local Electrode Atom Probe, or LEAP 3000XSi, allows for 3D atomic scale analysis of a variety of material. The 3000XSi was acquired through NSF-DMR-0722631

- FEI's Quanta 200 3D FIB/SEM with an Omniprobe 200 in-situ manipulator, EDAX EBSD/EDS Pegasus system, serial slice/view software, and variable pressure mode.

- TESCAN LYRA-3 XMU FIB-FESEM equipped with 5 in situ gas injection ports, micromanipulation and nano-lithographic patterning capability, and variable pressure mode.

- FEI's Tecnai F20 Super Twin 200kev FEG (S)TEM acquired through NSF-DMR -0421376. This (S)TEM is equipped with EDAX EDS, STEM imaging capability using a HAADF Detector, and both a high and low speed CCD cameras. Recent upgrades also include the integrated NanoMegas ASTAR platform for nanoscale grain-to-grain orientation mapping capability

- JEOL 7000 FESEM, FEG-SEM with Oxford X-MAX SDD EDS, WDS and EBSD detectors, STEM mode and Nabity E Beam lithography. Acquired through a NSF-DMR-0321180 grant.

- Philips XL30 SEM. A thermionic emission microscope with an EDAX EDS platform.

- JEOL 8600 Electron microprobe, equipped with five WDS detectors, Bruker XFlash SDD as part of an upgrade from Advanced Microbeam, as well as a Gatan cathode luminescence detector.

- Kratos AXIS 165 XPS, acquired through a NSF DMR-9512264 grant, monochromated Al and dual $\mathrm{Al} / \mathrm{Mg} \mathrm{x}$-ray sources, electron gun for auger, and an ion gun for etching and depth profiling.

- Bruker D8 Discover, with a HI-STAR 2D x-ray detector coupled with the GADDS system, Cu or Co X-ray sources, DIFFRAC ${ }^{\text {PLUS }}$ analysis suite, temperature control stage, and ICDD database.

- Ancillary specimen preparation equipment including a Fichione 1010 ion mill and 1020 plasma cleaner, Cressington coaters, dimple grinders and polishing equipment.

References

[1] http://caf.ua.edu/

[2] http://www.ua.edu/ 

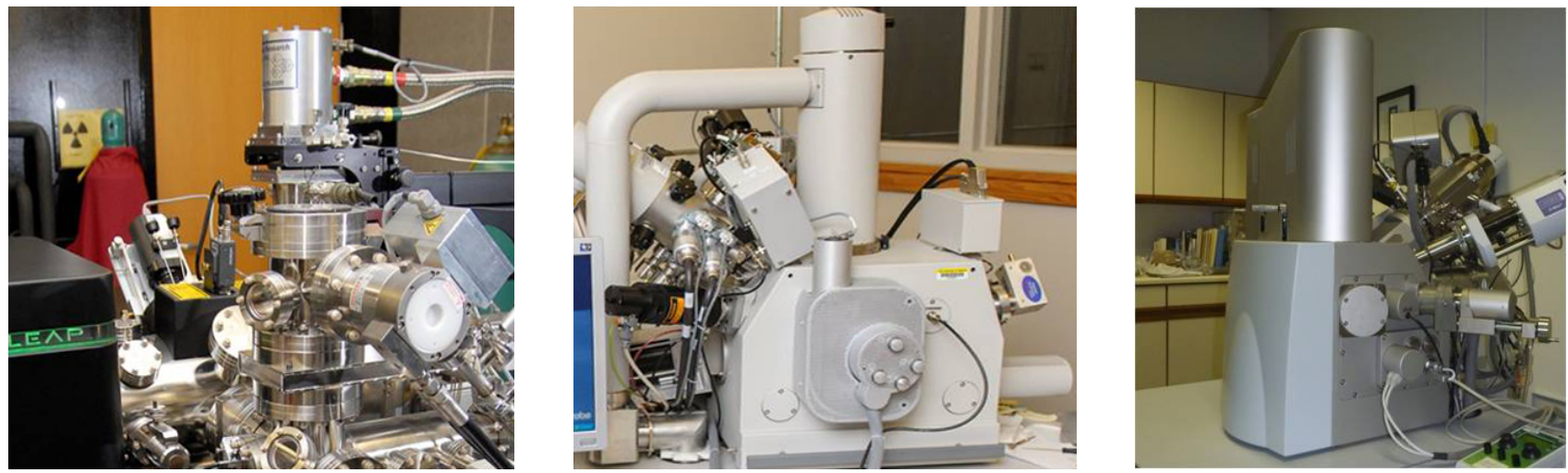

Fig. 1: (a) Cameca's LEAP 3000XSi. (b) FEI's Quanta 200 3D FIB/SEM. (c) TESCAN's LYRA-3 XMU FIB-FESEM
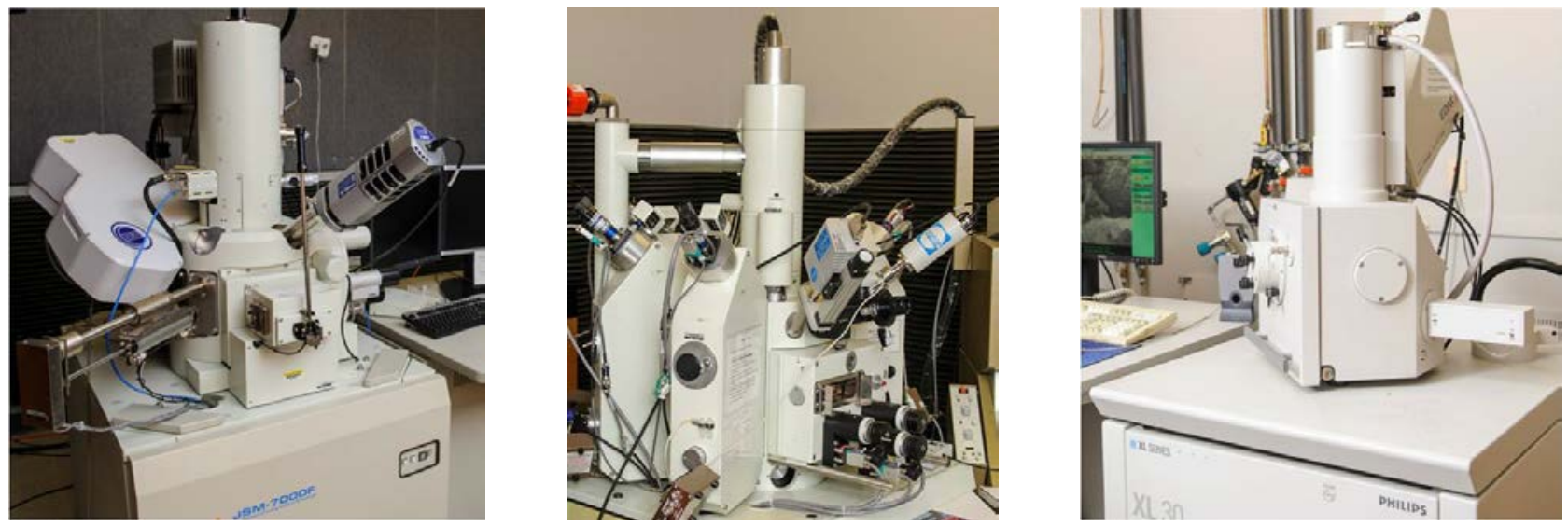

Fig. 2: (a) JEOL 7000 FESEM. (b) JEOL 8600 Electron Microprobe. (c) Phillips XL-30.
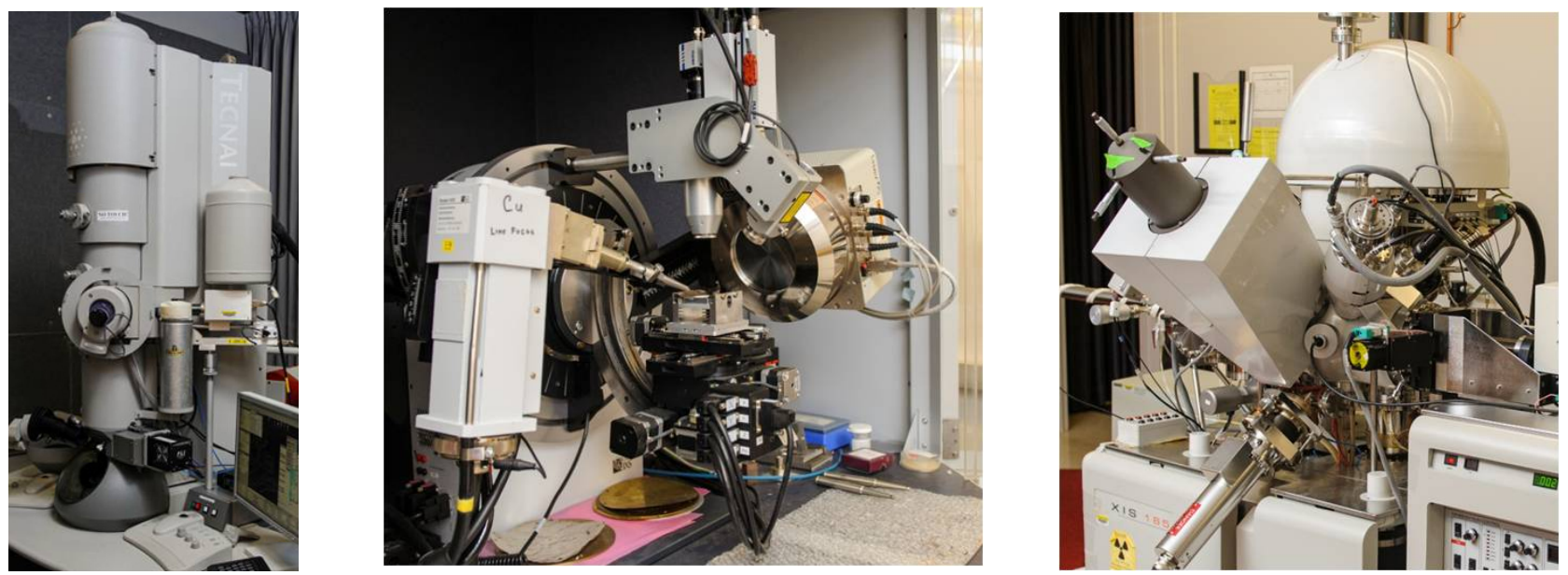

Fig. 3: (a) FEI's Tecnai F20 Super Twin 2200 Kev TEM. (b) Bruker D8 Discover. (c) Kratos AXIS 165 XPS 